PROCEEDINGS OF THE

AMERICAN MATHEMATICAL SOCIETY

Volume 132, Number 3, Pages 841-845

S 0002-9939(03)07092-8

Article electronically published on August 19, 2003

\title{
THE CAUCHY PROBLEM FOR A CLASS OF KOVALEVSKIAN PSEUDO-DIFFERENTIAL OPERATORS
}

\author{
ROSSELLA AGLIARDI AND MASSIMO CICOGNANI
}

(Communicated by David S. Tartakoff)

\begin{abstract}
We prove the $H^{\infty}$ well-posedness of the forward Cauchy problem for a pseudo-differential operator $P$ of order $m \geq 2$ with the Log-Lipschitz continuous symbol in the time variable. The characteristic roots $\lambda_{k}$ of $P$ are distinct and satisfy the necessary Lax-Mizohata condition $\operatorname{Im} \lambda_{k} \geq 0$. The LogLipschitz regularity has been tested as the optimal one for $H^{\infty}$ well-posedness in the case of second-order hyperbolic operators. Our main aim is to present a simple proof which needs only a little of the basic calculus of standard pseudodifferential operators.
\end{abstract}

\section{INTRODUCTION}

Let us consider the Cauchy problem

$$
\left\{\begin{array}{l}
P u(t, x)=f(t, x), \quad 0 \leq t \leq T, \quad x \in \mathbf{R}^{n} \\
\partial_{t}^{j} u(0, x)=g_{j}(x), \quad 0 \leq j \leq m-1
\end{array}\right.
$$

for a pseudo-differential operator of Kovalevskian type

$$
P=D_{t}^{m}+\sum_{j=0}^{m-1} A_{j}\left(t, x, D_{x}\right) D_{t}^{j}, \quad A_{j}(t) \in O P S^{m-j},
$$

of order $m \geq 2$ in $[0, T] \times \mathbf{R}^{n}$.

One says that problem (1.1) is well posed in the Sobolev space $H^{\infty}=H^{\infty}\left(\mathbf{R}^{n}\right)=$ $\bigcap H^{\mu}\left(\mathbf{R}^{n}\right)$ if for every $f \in \mathcal{C}\left([0, T] ; H^{\infty}\right)$ and $g_{j} \in H^{\infty}, 0 \leq j \leq m$, there is a $\mu$ unique solution $u \in \mathcal{C}^{m}\left([0, T] ; H^{\infty}\right)$.

In this paper we are concerned with the question of what kind of regularity in the time variable $t$ one has to assume for the $A_{j}$ 's in (1.2) in order to obtain such a well-posedness. From [1] and [2] we know that for second-order strictly hyperbolic differential operators the sharp regularity is the Log-Lipschitz continuity: a function $a(t)$ is said to be Log-Lipschitz continuous, in short $a \in L L([0, T])$, if it satisfies

$$
|a(t)-a(s)| \leq C|t-s||\log | t-s||, \quad 0<|t-s|<\frac{1}{2} .
$$

Received by the editors September 30, 2002 and, in revised form, November 5, 2002.

2000 Mathematics Subject Classification. Primary 35G10, 35L30.

Key words and phrases. Strictly hyperbolic operators, energy estimates, Log-Lipschitz continuity. 
Here we prove that $H^{\infty}$ well-posedness holds true for any operator of the type (1.2) with $A_{j}(t) \in L L\left([0, T] ; O P S^{m-j}\right), j=0, \ldots, m-1$, provided that the characteristic roots $\lambda_{k}(t, x, \xi)$ of $P$ are distinct and satisfy the necessary Lax-Mizohata condition

$$
\mathcal{I} m \lambda_{k}(t, x, \xi) \geq 0 \text { for large }|\xi|, k=1, \ldots, m .
$$

Our main aim is to give a simple proof which needs only basic calculus of classical pseudo-differential operators.

Notation. Throughout this paper, $x, \xi \in \mathbf{R}^{n},\langle\xi\rangle$ denotes $\sqrt{1+|\xi|^{2}}$ and $D_{x}=$ $-i \nabla_{x}$. Since it concerns the notation for pseudo-differential operators, we follow [3], which we refer to also for all the results we need.

Therefore, $S^{N}$ will denote the class of the symbols $a(x, \xi)$ such that

$$
\sup _{x, \xi}\left|\partial_{\xi}^{\alpha} D_{x}^{\beta} a(x, \xi)\right| \cdot\langle\xi\rangle^{|\alpha|-N}<\infty
$$

for every $\alpha, \beta \in \mathbf{N}^{n}$. Moreover, $S_{\ell o g}^{N}$ will denote the class of symbols with the following property:

$$
\sup _{x, \xi}\left|\partial_{\xi}^{\alpha} D_{x}^{\beta} a(x, \xi)\right| \cdot\langle\xi\rangle^{|\alpha|-N} / \log (1+\langle\xi\rangle)<\infty
$$

for every $\alpha, \beta \in \mathbf{N}^{n}$.

For symbols depending also on a time variable, we introduce the following notation: $a(t, x, \xi) \in L L\left([0, T] ; S^{N}\right)$, that is, $a(t, x, \xi)$ is Log-Lipschitz with respect to $t$, whenever for every $\alpha, \beta \in \mathbf{N}^{n}$ there exists $C_{\alpha, \beta}>0$ such that

$$
\sup _{x \in \mathbf{R}^{n}}\left|\partial_{\xi}^{\alpha} D_{x}^{\beta}(a(t, x, \xi)-a(s, x, \xi))\right| \leq C_{\alpha, \beta}|t-s||\log | t-s||\langle\xi\rangle^{N-|\alpha|},
$$

$0<|t-s|<1 / 2$.

The Cauchy problem. Let us consider an operator $P=P\left(t, x, D_{t}, D_{x}\right)$ in $[0, T] \times$ $\mathbf{R}^{n}$ given by

$$
P=D_{t}^{m}+\sum_{j=0}^{m-1} A_{j}\left(t, x, D_{x}\right) D_{t}^{j}
$$

where $A_{j} \in \mathcal{C}\left([0, T] ; S^{m-j}\right)$. Let $P^{0}=D_{t}^{m}+\sum_{j=0}^{m-1} A_{j}^{0}\left(t, x, D_{x}\right) D_{t}^{j}$ be such that

$$
A_{j}-A_{j}^{0} \in \mathcal{C}\left([0, T] ; S^{m-1-j}\right), \quad j=0, \ldots, m-1,
$$

and

$$
P^{0}(t, x, \tau, \xi)=\prod_{k=1}^{m}\left(\tau-\lambda_{k}(t, x, \xi)\right)
$$

with

$$
\lambda_{k} \in L L\left([0, T] ; S^{1}\right), \quad k=1, \ldots, m .
$$

We assume that the roots are distinct:

$$
\left|\lambda_{j}(t, x, \xi)-\lambda_{k}(t, x, \xi)\right| \geq c|\xi| \text { for large }|\xi|, j \neq k, c>0,
$$


and that they fulfill the Lax-Mizohata condition:

$$
\mathcal{I} m \lambda_{k}(t, x, \xi) \geq 0 \text { for large }|\xi|
$$

Remark 3.1. If (3.2) holds for $A_{j}^{0}$ such that each function $A_{j}^{0}(t, x, \xi)$ is homogeneous of degree $m-j$ in $\xi$ for large $|\xi|$, i.e. $A_{j}^{0}(t, x, \theta \xi)=\theta^{m-j} A_{j}^{0}(t, x, \xi), \theta \geq 1,|\xi| \geq M$, and we assume

$$
A_{j}^{0} \in L L\left([0, T] ; S^{m-j}\right), j=0, \ldots, m-1,
$$

then, after a modification in a neighborhood of $\xi=0$, we have (3.4), in view of $(3.5)$.

Our main result is that the Cauchy problem

$$
\begin{cases}P u(t, x)=f(t, x), & 0 \leq t \leq T \\ D_{t}^{j} u(0, x)=g_{j}(x), & 0 \leq j \leq m-1,\end{cases}
$$

is well posed in $H^{\infty}$ (with a loss of derivatives). In fact, we have the following.

Theorem 3.1. Let $P$ satisfy (3.2), (3.4), (3.5) and (3.6). Then there is $\delta>0$ such that for every $\mu \in \mathbf{R}$, every $f \in \mathcal{C}\left([0, T] ; H^{\mu}\right)$ and $g_{j} \in H^{\mu+m-j-1}, 0 \leq j \leq m-1$, the Cauchy problem (3.7) has a unique solution $u \in \bigcap_{j=0}^{m-1} \mathcal{C}^{j}\left([0, T] ; H^{\mu-\delta T+m-j-1}\right)$. The solution satisfies the inequality$$
\sum_{j=0}^{m-1}\left\|\partial_{t}^{j} u(t)\right\|_{\mu-\delta t+m-j-1}^{2} \leq C\left\{\sum_{j=0}^{m-1}\left\|g_{j}\right\|_{\mu+m-j-1}^{2}+\int_{0}^{t}\|f(s)\|_{\mu-\delta s}^{2} d s\right\}, 0 \leq t \leq T,
$$

for some $C=C_{\mu}>0$.

Proof. The first step is to carry the factorization (3.3) to the operator level. Let us introduce the following regularization of $\lambda_{k}$ with respect to the variable $t$ :

$$
\tilde{\lambda}_{k}(t, x, \xi)=\int \lambda_{k}(s, x, \xi) \rho((t-s)\langle\xi\rangle)\langle\xi\rangle d s,
$$

where $\rho \in \mathcal{C}_{0}^{\infty}(\mathbf{R}), 0 \leq \rho \leq 1, \int \rho=1$ and we have set $\lambda_{k}(s, x, \xi)=\lambda_{k}(T, x, \xi)$ for $s>T$ and $\lambda_{k}(s, x, \xi)=\lambda_{k}(0, x, \xi)$ for $s<0$. It is easy to see that

$$
\begin{aligned}
& \tilde{\lambda}_{k} \in \mathcal{C}\left([0, T] ; S^{1}\right), \\
& \tilde{\lambda}_{k}-\lambda_{k} \in \mathcal{C}\left([0, T] ; S_{\ell o g}^{0}\right) \text {, }
\end{aligned}
$$

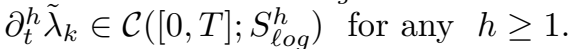

Thus the operator $P$ can be written in the form

$$
P=\left(D_{t}-\tilde{\lambda}_{m}\left(t, x, D_{x}\right)\right) \ldots\left(D_{t}-\tilde{\lambda}_{1}\left(t, x, D_{x}\right)\right)+\sum_{j=0}^{m-1} R_{j}\left(t, x, D_{x}\right) D_{t}^{j}
$$


with $R_{j} \in \mathcal{C}\left([0, T] ; S_{\ell o g}^{m-j-1)}\right)$. Next we want to reduce the scalar equation $P u=f$ to an $m \times m$ system $\mathcal{L U}=\mathcal{F}$. Let us define $\mathcal{U}={ }^{t}\left(u_{0}, \ldots, u_{m-1}\right)$ by

$$
\begin{aligned}
& u_{0}=\left\langle D_{x}\right\rangle^{m-1} u, \\
& u_{1}=\left\langle D_{x}\right\rangle^{m-2}\left(D_{t}-\tilde{\lambda}_{1}\left(t, x, D_{x}\right)\right) u, \\
& \cdots \ldots . \\
& u_{m-1}=\left(D_{t}-\tilde{\lambda}_{m-1}\left(t, x, D_{x}\right)\right) \ldots\left(D_{t}-\tilde{\lambda}_{1}\left(t, x, D_{x}\right)\right) u .
\end{aligned}
$$

Denoting ${ }^{t}\left(\left\langle D_{x}\right\rangle^{m-1} u,\left\langle D_{x}\right\rangle^{m-2} D_{t} u, \ldots, D_{t}^{m-1} u\right)$ by $\mathcal{V}$, we immediately have

$$
\mathcal{U}=Q\left(t, x, D_{x}\right) \mathcal{V}, \quad \mathcal{V}=Q_{0}\left(t, x, D_{x}\right) \mathcal{U},
$$

where the symbols of the entries of the $m \times m$ matrices $Q$ and $Q_{0}$ belong to $\mathcal{C}\left([0, T] ; S^{0}\right)$. Thus the equation $P u=f$ is equivalent to an $m \times m$ system

$$
\mathcal{L U}=\mathcal{F},
$$

where $\mathcal{F}={ }^{t}(0, \ldots, 0$, if $)$ and

$$
\mathcal{L}=\partial_{t}-i \Lambda\left(t, x, D_{x}\right)+B\left(t, x, D_{x}\right)
$$

with

$$
\Lambda=\left[\begin{array}{cccc}
\tilde{\lambda}_{1}\left\langle D_{x}\right\rangle & \ldots & \ldots & 0 \\
0 & \ldots & \ldots & 0 \\
0 & \ldots & \tilde{\lambda}_{m-1}\left\langle D_{x}\right\rangle & 0 \\
0 & \ldots & \ldots & \tilde{\lambda}_{m}
\end{array}\right]
$$

and the entries of $B(t, x, \xi)$ in $\mathcal{C}\left([0, T] ; S_{\ell o g}^{0}\right)$. The operator $\Lambda$ can be diagonalized by means of

$$
M=\left[\begin{array}{ccc}
1 & & d_{i j} \\
& \ddots & \\
0 & & 1
\end{array}\right], \quad d_{i j}(t, x, \xi)=\langle\xi\rangle^{j-1} / \prod_{k=i}^{j-1}\left(\tilde{\lambda}_{j}-\tilde{\lambda}_{k}\right), i<j \text {, for large }|\xi| .
$$

From (3.10) we have $M, M^{-1} \in \mathcal{C}\left([0, T] ; S^{0}\right), \partial_{t} M, \partial_{t} M^{-1} \in \mathcal{C}\left([0, T] ; S_{\ell o g}^{0}\right)$. Thus, we have

$$
\mathcal{L}_{1}:=M^{-1} \mathcal{L} M=\partial_{t}-i \Delta\left(t, x, D_{x}\right)+B_{1}\left(t, x, D_{x}\right),
$$

where $\Delta$ is the diagonal matrix of the $\tilde{\lambda}_{k}$ 's and $B_{1} \in \mathcal{C}\left([0, T] ; S_{\ell o g}^{0}\right)$. Now, for $\mu \in \mathbf{R}$ and $\delta>0$ let us define the operator $\mathcal{L}_{2}=\left\langle D_{x}\right\rangle^{\mu-\delta t} \mathcal{L}_{1}\left\langle D_{x}\right\rangle^{-\mu+\delta t}$. We have

$$
\mathcal{L}_{2}=\partial_{t}-i \Delta\left(t, x, D_{x}\right)+B_{1}\left(t, x, D_{x}\right)+\delta \log \left\langle D_{x}\right\rangle I+B_{2}\left(t, x, D_{x}\right),
$$

where $B_{2} \in \mathcal{C}\left([0, T] ; S^{0}\right)$.

It remains to prove the following.

Proposition 3.2. It is possible to fix $\delta>0$ such that the Cauchy problem

$$
\left\{\begin{array}{l}
\mathcal{L}_{2} \mathcal{U}(t, x)=F(t, x), \quad 0 \leq t \leq T \\
\mathcal{U}(0, x)=G(x)
\end{array}\right.
$$


has a unique solution $\mathcal{U} \in \mathcal{C}\left([0, T] ; H^{1}\right) \cap \mathcal{C}^{1}\left([0, T] ; H^{0}\right)$ for any given $F \in \mathcal{C}([0, T]$; $\left.H^{1}\right)$ and $G \in H^{1}$. The solution satisfies the energy inequality

$$
\|\mathcal{U}(t)\|_{0}^{2} \leq C\left(\|\mathcal{U}(0)\|_{0}^{2}+\int_{0}^{t}\|\mathcal{F}(s)\|_{0}^{2} d s\right), \quad 0 \leq t \leq T,
$$

for some $C=C_{\mu}>0$.

Proof. We have only to prove that it is possible to fix $\delta>0$ such that

$$
\|\mathcal{U}(t)\|_{0}^{2} \leq C\left(\|\mathcal{U}(0)\|_{0}^{2}+\int_{0}^{t}\left\|\mathcal{L}_{2} \mathcal{U}(s)\right\|_{0}^{2} d s\right), \quad 0 \leq t \leq T,
$$

for every $\mathcal{U} \in \mathcal{C}\left([0, T] ; H^{1}\right) \cap \mathcal{C}^{1}\left([0, T] ; H^{0}\right)$, with a constant $C>0$ depending only on the seminorms of the symbols of $\Delta, B_{1}, B_{2}$ (hence depending on $\mu$ because of $B_{2}$ ). Then one can apply the usual energy method to solve (3.19), e.g. [3], pp. 236-240. To prove (3.21), we begin by choosing $\delta$ large enough in order to have positive operators $B_{1}\left(t, x, D_{x}\right)+\delta \log \left\langle D_{x}\right\rangle I$ in (3.18) for $t \in[0, T]$. This is possible from $B_{1} \in \mathcal{C}\left([0, T] ; S_{\text {log }}^{0}\right)$. So, we obtain

$$
\begin{aligned}
& \frac{d}{d t}\|\mathcal{U}(t)\|_{0}^{2} \leq 2 \mathcal{R} e\left(i \Delta \mathcal{U}(t)-B_{2} \mathcal{U}(t)+\mathcal{L}_{2} \mathcal{U}, \mathcal{U}(t)\right) \\
& \leq 2 \mathcal{R} e(i \Delta \mathcal{U}(t), \mathcal{U}(t))+C_{\mu}\left(\|\mathcal{U}(t)\|_{0}^{2}+\left\|\mathcal{L}_{2} \mathcal{U}(t)\right\|_{0}^{2}\right)
\end{aligned}
$$

Then from (3.6) we can apply the sharp Gårding inequality to the first-order operator $-i \Delta$ :

$$
2 \mathcal{R} e(-i \Delta \mathcal{U}(t), \mathcal{U}(t)) \geq-C\|\mathcal{U}(t)\|_{0}^{2}, \quad C>0
$$

which gives

$$
\frac{d}{d t}\|\mathcal{U}(t)\|_{0}^{2} \leq C_{\mu}^{\prime}\left(\|\mathcal{U}(t)\|_{0}^{2}+\left\|\mathcal{L}_{2} \mathcal{U}(t)\right\|_{0}^{2}\right) .
$$

Gronwall's inequality yields (3.21), completing the proof.

\section{REFERENCES}

[1] F. Colombini, E. De Giorgi, and S. Spagnolo, Sur les équations hyperboliques avec des coefficients qui ne dépendent que du temps, Ann. Scuola Norm. Sup. Pisa 6 (1979), 511-559. MR 81c:35077

[2] F. Colombini and N. Lerner, Hyperbolic operators with non-Lipschitz coefficients, Duke Math. J. 77 (1995), no. 3, 657-698. MR 96d:35075

[3] H. Kumano-go, Pseudodifferential operators, The MIT Press, Cambridge, Massachusetts, and London, England, 1981. MR 84c:35113

University of Ferrara, via Machiavelli 35, 44100 Ferrara, Italy

E-mail address: agl@dns.unife.it

University of Bologna, via Genova 181, 47023 Cesena, Italy

E-mail address: cicognan@dm.unibo.it 